\title{
Special features of obtaining fine powders for additive technologies
}

\author{
Elena Matys ${ }^{1, *}$, Yury Deniskin ${ }^{2}$, Ekaterina Stativa $^{3}$ and Dmitriy Shlychkov ${ }^{4}$ \\ ${ }^{1}$ Tyumen Industrial University, Volodarskogo str., 38, Tyumen, 625000, Russia \\ ${ }^{2}$ Moscow Aviation Institute, Volokolamskoe highway, 4, Moscow, 125993, Russia \\ ${ }^{3}$ Moscow State University of Civil Engineering, 129337, 26, Yaroslavskoe Shosse, Moscow, Russia \\ ${ }^{4}$ Joint stock company «Mosgaz», 1/11, Mruzovsky lane, Moscow, 105120, Russia
}

\begin{abstract}
The paper discusses some issues of fine powders production technologies adjustment. These powders can be used in concrete mixtures for $3 \mathrm{D}$ building printers. The work proposes the implementation of vibrational sieves which use the dry method of sifting fine and ultra-fine powders with high performance and efficiency. In this technology, ground material is removed from the mill which prevents the material from consolidation and the grinding process from the slowdown. The paper focuses on a new type of equipment able to withstand overload conditions of up to $30 \mathrm{~g}$. It is lightweight, compact, it does not require filters, cyclones, high-pressure fans, sluice feeders. Its sieves do not clog and do not require tightening which considerably simplifies and reduces the cost of operation.
\end{abstract}

\section{Introduction}

The development of new construction technologies poses new challenges for developers. Just like that, 3D building printers are under development all over the world and Russian equipment that allows erecting multi-storey buildings have been introduced as well. However, the optimization of the compositions of construction mixtures in terms of price and quality is still underway [1]. There are special requirements that apply to the materials and they differ from ones introduced in conventional formwork technologies. One of such requirements is the high speed of achieving the nominal strength of the concrete, as the new layers should not crush the underlying. Research has found that the additives of mechanically activated powders in the composition of concrete significantly change its properties. Regular concrete gains $16 \%$ of its strength in 24 hours, while one with a finely dispersed aggregate reaches this strength in 7 hours in equal conditions [2-4].

The issues of finely dispersed additives are discussed also in papers [5, 6].

Mechanical activation for building materials was done back in time using technologies of the Tallinn scientist Johannes Hint. At that time, a lot of research was conducted on the selection of grinding equipment, taking into account the features of the strengthening of building blocks. The results presented that the traditional equipment such as ball mills, for example, does not provide a sufficient degree of activation, despite the continued grinding.

\footnotetext{
*Corresponding author: e.matys@yandex.ru
} 
Accordingly, the implementation of a high-speed disintegrator was recommended. However, the subsequent wide use of disintegrators detected the rapid wear of the fingers in the baskets, especially when grinding abrasive materials.

Planetary ball mills with power density have demonstrated significant results, nevertheless, they have not proved themselves suitable for industrial application. The reason for that being that they do not provide efficient continuous high performance due to the unreliability of their components operating under high overload and rapid wear linings.

Vibration mills impose significant vibration load on their foundations and adjacent equipment. Therefore, there is the task to find a way to use a new set of reliable working equipment for powder activation. Nevertheless, ball mills have proved themselves to be the most reliable grinding equipment.

\section{Methods}

The inductive method was used in writing the work. It includes, first of all, the mandatory accumulation of experimental information, which acts as a basis for further generalizations. Ball mills are the most widely used and reliable grinding equipment. Paper [7] compares the performance of a laboratory Pulverisette planetary mill for 4 minutes and of a ball mill for only 15 minutes. As a result, the planetary mill was considered to be more efficient. Even with an overload condition of $10 \mathrm{~g}$, the unit power of the planetary mill increases depending linearly on the overload and depending on the increase of the critical speed to the 0.5 power, e.i. 31.6 times. To provide a more accurate assessment, it is necessary to significantly prolong the processing in the ball mill while using tools that prevent grinding.

Ball mills are less power dense, although are large and therefore, have a longer wear time of linings and are easy to operate, its liners and lifters do not require replacement for a few months of operation. At the same time, the planetary mill under intensive load requires replacement of the liners in a few days of operation and its bearings placed in a powerful centrifugal field can quickly fail to function.

The reason being that the grinding in the mill induces the wear of liners and lifters in proportion to the volume of processed feedstock. Wear levels can be as high as one kilogram of metal per tonne of processed material. Considering the fact that the total weight of the lining of the ball mill is tens of tons, and of the planetary mill is tens of kilograms, the need for constant maintenance of power dense equipment becomes inevitable. A common problem of ball mills operating using dry method is the consolidation of the crushed material after some grinding time. This aggregation process takes significant grinding energy and reduces the free surface of the ground material. Therefore, it is very important to remove the crushed material from the mill in time to prevent it from being involved in the process of consolidation. It was established experimentally that the accumulation of fine fraction of the material the grinding process slows drastically due to the dry pressing of the material in which the balls even get stuck [8]. To continue the grinding process, the activation of the processes of moving the balls and the material is used, e.g. the inclination of the rotation axis of the mill, the increase of the speed of circulation of the crushed material, the use of spiral lifters, etc. [8-10].

The most effective process that eliminates the inhibition of grinding is the process of removing the finished product from the mill, which does not allow it to aggregate and press in the mill. To complete this task, highly efficient classificatory equipment is needed.

Essentially, complexes of aerial classification are applied, since the sifting of fine materials through traditional screens using dry method is not possible because of the rapid clogging of the mesh [9]. Nevertheless, there are plenty of intensely wearing components in aerial classification systems. Not only that, material processing consumes large quantities 
of energy, filters are being clogged which increases the resistance of air ducts, leads to sluice feeders wear out.

The example of such a system is the complex LF-CM 1000 of large volume and area and with an installed capacity of $22 \mathrm{~kW}$. The height of the system is $3.4 \mathrm{~m}$ the weight is $1250 \mathrm{~kg}$. The efficiency of air classificatory equipment usually does not exceed $70 \%$ and it is difficult to improve its performance. To do so, the creation of a system of multiple repurifications is needed, with an inevitable increase of pieces of the installation. Besides that, there is a chance of coarse particles entering fine particles reservoir, as the system does not render mechanical calibration possible in contrast to the mesh system. The complex LFCM 1000 is presented in figure 1.

\section{Results}

However, in St. Petersburg a technique was developed that allows sifting fine powders with the fineness of up to 20 microns, with a capacity of hundreds of $\mathrm{kg}$ per $\mathrm{h}$ with high efficiency and with a power consumption of vibrators of up to 600 watts. The equipment allows for the sifting of the materials that revoke multiple challenges using the conventional methods of material processing. The system is capable of sifting materials of different shapes, e.g. flat particles of phlogopite, powders with a low specific weight, e.g., microspheres, viscous substances based on different types of resin and other materials. These kinds of materials, namely, binding, reinforcing powders are of specific interest for the production of initial additives for the manufacturing of fast-hardening concrete used for building 3D printers. A fragment of the manufacturer's performance data is presented in table 1 .

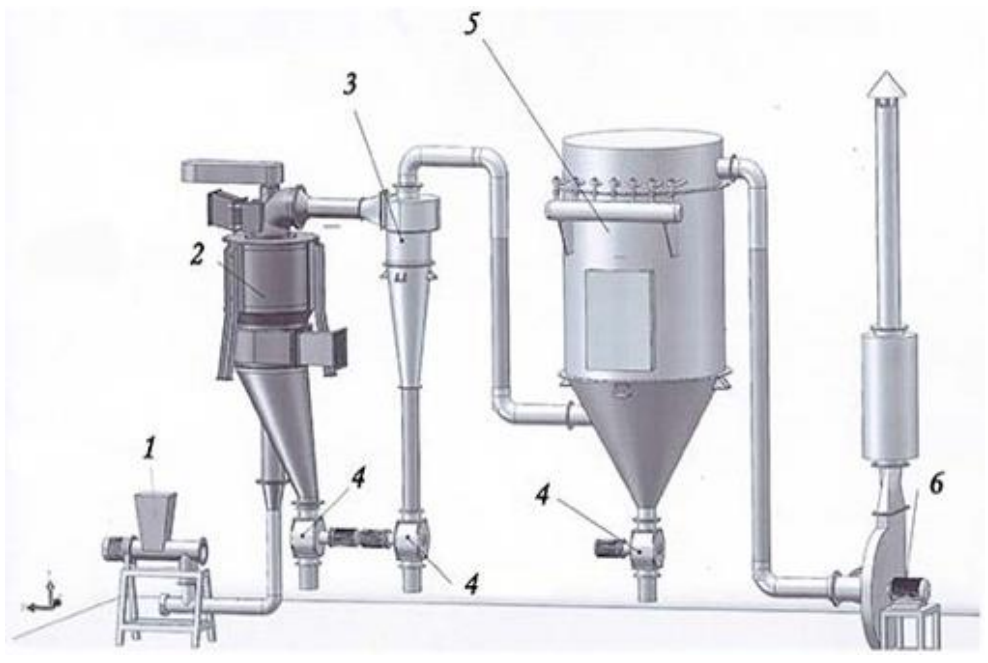

Fig. 1. Complex LF-CM 1000, where: 1. Receiving bin; 2. Aerial centrifugal classifier; 3. Cyclone collector; 4. Sluice feeder; 5 . Air filter; 6 . High-pressure fan.

The general arrangement of the MV1-APM-1.0 vibrational sieve with a mesh square of $1 \mathrm{~m}^{2}$ and a weight of $350 \mathrm{~kg}$, with overall dimensions of 1360x1050x1055 (length $\mathrm{x}$ width $\mathrm{x}$ height, $\mathrm{mm}$ ) is presented in Figure 2. 


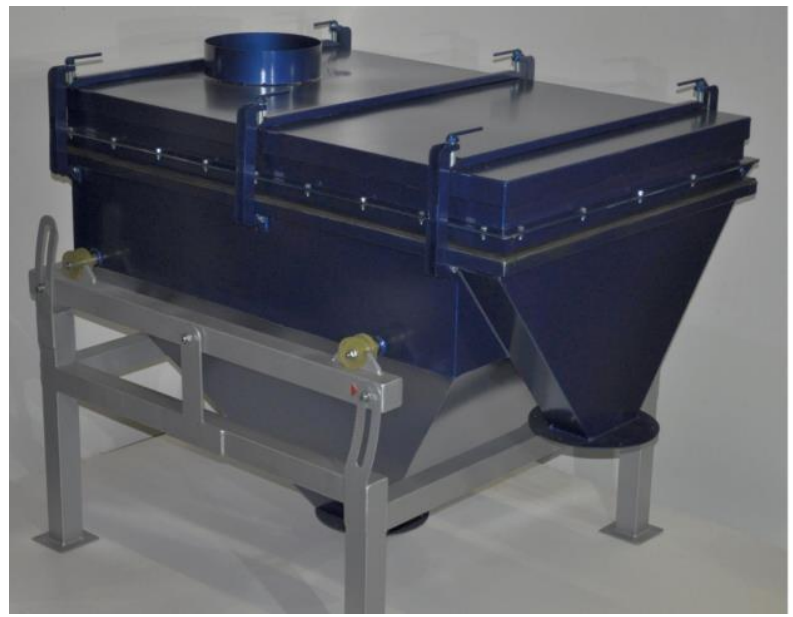

Fig. 2. Vibrational sieve MV1- APM-1.0.

Table 1. Manufacturer's performance data.

\begin{tabular}{|l|l|l|}
\hline Name of the material & $\begin{array}{l}\text { Fineness, } \\
\mathrm{mm}\end{array}$ & $\begin{array}{l}\text { Performance per } 1 \mathrm{~m}^{2} \text { (with an efficiency of more than } \\
85 \%\end{array}$ \\
\hline Diamond dust & 0.074 & 650 \\
\hline Alginate & 0.12 & 260 \\
\hline Alpha-alumina & 0.063 & 300 \\
\hline & 0.046 & 190 \\
Aluminium powder PA-3 & 0.065 & 245 \\
& 0.074 & 360 \\
& 0.1 & 460 \\
& 0.16 & 670 \\
\hline Aluminium powder PAP- & 0.046 & 410 \\
\hline
\end{tabular}

The vibrational sieve was installed successfully into the chain with a ball mill and the circulation of the dry material with the fineness of 40 microns was maintained. A new result was obtained by the combination of a traditional mill and a new type of equipment able to withstand overload conditions of up to $30 \mathrm{~g}$.

It is lightweight, compact, it does not require filters, cyclones, high-pressure fans, sluice feeders. Its sieves do not clog and do not require tightening which considerably simplifies and reduces the cost of operation. The sieve is made sealed, so there is no dust. A small amount of material accumulates on the bottom of the leaks on the vibrators, but not during the working process.

To change the efficiency of sieving, the sieve is made with the option of setting the angle between the plane of the upper cover of the vibrating screen and the plane of the horizon. The level of vibration and noise is much lower than on traditional screens due to the screen body being stationary. 


\section{Conclusions}

To conclude, the combination of traditional proven equipment, such as reliable and timetested ball mills with new classificatory sieves, allowing to screen out the fine fraction of the crushed material, opens up new opportunities for the production of ultra-fine powders, among others ones for additive technologies.

The implementation of small screens which does not clog and does not require tightening in the circulation of dry materials from the mill, as well as the restoration of coarse particles in the mill allows to avoid aggregation and pressing of the final material and to prevent the grinding process from the slowdown. A high degree of material classification of up to $95 \%$ can be achieved.

\section{Reference}

1. M.A. Zlenko, A.A. Popovich, I.N. Mutilin, Additive technologies in mechanical engineering (Polytechnic University, St. Petersburg, 2013)

2. Y.M. Bazhenov, Technology of concrete (Publishing ASV, Moscow, 2002)

3. I.N. Akhverdov, Basic physics of concrete (Stroyizdat, Moscow, 1981)

4. A.N. Ivanov-Gorodov, The influence of grain composition of Portland cement in its building and technical properties, Dissertation (Moscow, 1960)

5. A.A. Lushnikova, M. Sokovikova, I. Pudov, Vestnik SUSU 16, 30-33 (2011)

6. T.Z. Panina, A.A. Lygina, M. Gubaidulina, K.G. Nikolayev, A.N. Khalitova, Izvestiya kgasu 4(22), 326-331 (2012)

7. O.N. Burenina, N.N. Davydova, A.V. Andreeva, M.E. Savvinova, The scientific journal of the Kuban state agrarian University 111(07) (2015)

8. I.I. Blekhman, Theory of vibration processes and devices (Publishing "Ore and Metals", SPb, 2013) ISBN 978-5-98191-074-6.

9. A.A. Heravi, O. Smirnova, V. Mechtcherine, RILEM Bookseries, 15, 266-274 (2018)

10. O.M. Smirnova, D.A. Potyomkin, International Journal of Civil Engineering and Technology (IJCIET). 9, Issue 7, 874-880. (2018) 